\title{
VALIDITY AND RELIABILITY OF INERTIAL MEASUREMENT UNITS FOR JUMP HEIGHT ESTIMATIONS: A SYSTEMATIC REVIEW
}

review paper

() Wroclaw University of Health and Sport Sciences

DOI: https://doi.org/10.5114/hm.2023.111548

\section{FILIPE MANUEL CLEMENTE ${ }^{1,2,3}$, GEORGIAN BADICU ${ }^{4}$, UDAY CH. HASAN ${ }^{5}$, ZEKI AKYILDIZ $^{6}$, JOSÉ PINO-ORTEGA ${ }^{7}$, RUI SILVA ${ }^{1}$, MARKEL RICO-GONZÁLEZ ${ }^{8}$}

${ }^{1}$ Escola Superior Desporto e Lazer, Instituto Politécnico de Viana do Castelo, Viana do Castelo, Portugal

${ }^{2}$ Research Center in Sports Performance, Recreation, Innovation and Technology, Melgaço, Portugal

${ }^{3}$ Instituto de Telecomunicações, Delegação da Covilhã, Covilhã, Portugal

${ }^{4}$ Department of Physical Education and Special Motricity, Transilvania University of Brasov, Brasov, Romania

${ }^{5}$ Department of Physical Education and Sport Science, Al-Kitab University, Kirkuk, Iraq

${ }^{6}$ Faculty of Sport Sciences, Gazi University, Ankara, Turkey

${ }^{7}$ Faculty of Sports Sciences, University of Murcia, San Javier, Spain

${ }^{8}$ Department of Physical Education and Sport, University of the Basque Country, Vitoria-Gasteiz, Spain

\section{ABSTRACT}

Inertial measurement units (IMUs) have been extensively used for measuring human motion. One particular outcome of interest in sports is vertical jump height, which is assessed in specific performance tests or actions occurring in training or match scenarios. This systematic review aimed to (1) identify and summarize studies that have examined the validity of wearable wireless IMUs for measuring jump height and (2) identify and summarize studies that have examined the reliability of wearable wireless IMUs for measuring jump height. A systematic review of the Cochrane Library, EBSCO, PubMed, SPORTDiscus, and Web of Science databases was performed in accordance with the Preferred Reporting Items for Systematic Reviews and Meta-Analyses guidelines. From the 596 studies initially identified, 30 were fully reviewed, and their outcome measures were extracted and analysed. Among the 16 different IMU models, 15 were considered valid, with only one device not validated. Of the 7 IMUs that were tested for reliability, all were considered reliable for measuring jump height. In general, however, despite these findings, IMUs are not considered accurate enough to detect small changes in performance. Also, generalizations were not possible for athletic populations given the lack of studies with such samples.

Key words: inertial measurement units, jump height, sports technology, micro-electro-mechanical systems, sensors, performance

\section{Introduction}

The measurement of vertical jumping performance has become one of the most popular strength and conditioning tests used in periodic assessment of athletic ability [1] and monitoring the readiness [2] of athletes in different sports. Among the numerous possibilities (e.g., drop jump, Abalakov test, Sargent jump test), squat jump (SJ) and countermovement jump (CMJ) are the most common [3, 4]. Both of these jump tests have the same objective: to perform the highest jump.
Different outcomes can be analysed while performing the jumping tests (e.g., power, force, velocity, impulse) [1]. Thus, jump height is the most commonly used jump measure in the sports context [5]. However, it has been reported that jump peak velocity is a better method to track changes over time than mean power and height [6].

Different systems have been employed for measuring jump height, of which the most common are: (i) contact platforms (contact mat) [7]; (ii) infrared platforms [8]; (iii) force platforms [9]; (iv) video-based

Correspondence address: Filipe Manuel Clemente, Escola Superior Desporto e Lazer, Instituto Politécnico de Viana do Castelo, Rua Escola Industrial e Comercial de Nun'Álvares, 4900-347 Viana do Castelo, Portugal, e-mail: filipe.clemente5@gmail.com, https://orcid.org/0000-0001-9813-2842

Received: September 7, 2021

Accepted for publication: September 30, 2021

Citation: Clemente FM, Badicu G, Hasan UCh, Akyildiz Z, Pino-Ortega J, Silva R, Rico-González M. Validity and reliability of inertial measurement units for jump height estimations: a systematic review. Hum Mov. 2022;23(4):1-20; doi: https:// doi.org/10.5114/hm.2023.111548. 
F.M. Clemente et al., Inertial measurement units for jump height estimations

methods [5]; (v) linear position transducers [10]; and (vi) inertial measurement units (IMUs) [11]. Among these systems, the force platform has been considered the gold standard equipment for assessing jump measures [12]. Although force platforms have an easy portability, only a few can be used without constrained settings, since such equipment requires the athlete to be in a specific location (above the platform).

In addition, video-based methods are limited to only a few athletes or a single athlete in some cases. Thus, IMUs constitute a useful training tool, since athletes can use them in different contexts. For instance, IMUs can be applied to monitor sleep quality [13] and barbell velocity during strength and power training [14], as well as to detect changes of direction [15], apart from other uses [16]. Therefore, more than simply being used to assess vertical jump height, IMUs are also a suitable instrument for external load monitoring in sports that present a high volume of jumps, such as volleyball [17]. In fact, some studies have tested the validity and reliability of IMU devices to quantify the number of jumps during training and competition in indoor team sports $[18,19]$. In outdoor sports, elite teams commonly quantify the external load measures using global positioning systems (GPS) that have accelerometers, gyroscopes, and magnetometers housed in each unit [20]. Furthermore, IMUs can be utilized in weight room settings to monitor barbell velocities during gym training known as velocity-based training [14].

IMU is a type of micro-electro-mechanical system technology that can integrate 3 types of sensors: (i) accelerometers, (ii) gyroscopes, and (iii) magnetic sensors. It is mostly used for measuring velocity, orientation, and gravitational force [21]. While the accelerometers detect inertial acceleration, the gyroscope measures the angular rotation [21] and the magnetometers are used for orientation [16]. The use of such devices would allow coaches and practitioners to save time, given that the apparatus to quantify the external loads would simultaneously assess jump height, as typically IMUs have accelerometers, gyroscopes, and magnetometers integrated in the device. Thus, most IMUs offer real-time data analysis and are usually connected to a smartphone or other electronic device, such as a tablet [22, 23]. However, further statistical analysis for ensuring better communication and decision-making must be done [24].

Given that these types of instruments are used in various sports settings, coaches and practitioners would benefit from applying a device that has the potential to measure different aspects of training, such as external loads and periodic and readiness-to-train jump assess- ments. However, for external load monitoring and vertical jump evaluation, the accuracy and precision of the measuring systems must be ensured so that the inferences obtained from assessments are accurate. Otherwise, the perceived changes in performance may be related to the error and variability of the instrument, not the player's actual performance. Therefore, original reports of the validity and reliability of IMUs have been disseminated in different contexts and with different jump assessment protocols [11, 12, 17, 25, 26].

Despite the existence of systematic reviews about wearable IMUs in sports [16, 27], to the authors' knowledge, there are no systematic reviews that focus on the reliability and validity of wearable IMUs for measuring outcomes such as vertical jump height. There is a need to properly identify the validity and reliability of IMUs for measuring vertical jump height. Such a review would provide sports scientists and coaches with valuable information for interpreting human performance with the use of these systems. Therefore, the aim of this systematic review was twofold: (1) to identify and summarize studies that have examined the validity of wearable wireless IMUs for measuring jump height and (2) to identify and summarize studies that have examined the reliability of wearable wireless IMUs for measuring jump height.

\section{Material and methods}

The systematic review strategy was implemented in accordance with the Preferred Reporting Items for Systematic Reviews and Meta-Analyses (PRISMA) guidelines [28]. The protocol was registered in the International Platform of Registered Systematic Review and Meta-Analysis Protocols with the number 2020120134 and the DOI number 10.37766/inplasy 2020.12.0134.

\section{Eligibility criteria}

The inclusion criteria can be found in Table 1 .

The screening of the title, abstract, and reference list of each study to locate potentially relevant studies was independently performed by 2 of the authors (FMC and MRG). Additionally, they reviewed the full versions of the included papers in detail to identify articles that met the selection criteria. A further search within the reference lists of the included records was conducted to retrieve additional relevant studies. In the cases of discrepancies regarding the selection process, a discussion ensued with a third author (RS). Possible errata for the included articles were considered. 
Table 1. Inclusion criteria

\begin{tabular}{l}
\hline Inclusion criteria \\
\hline Test of a wearable wireless inertial measurement unit \\
\hline $\begin{array}{l}\text { Tests were conducted in humans in laboratory or field- } \\
\text { based conditions }\end{array}$ \\
\hline Estimation of jump height \\
\hline In the case of validity, the inertial measurement unit \\
was compared with a gold standard method (e.g., force \\
platform, contact platform, infrared platform, video- \\
based method) \\
\hline $\begin{array}{l}\text { In the case of validity, one of the following measures } \\
\text { were included: (i) typical error; (ii) mean absolute error; } \\
\text { (iii) correlation coefficient; (iv) standard error of the } \\
\text { estimate }\end{array}$
\end{tabular}

In the case of reliability, one of the following measures were included: (i) intraclass correlation test; (ii) coefficient of variation; (iii) standardized typical error; (iv) standard error of measurement

Only original and full-text studies written in English

\section{Information sources and search}

Electronic databases (Cochrane Library, EBSCO, PubMed, SPORTDiscus, and Web of Science) were searched for relevant publications prior to January 1 , 2021. The Web of Science was searched with filtering by "sport sciences". Keywords and synonyms were entered in various combinations in the title, abstract, or keywords: (sport* OR exercise* OR "physical activity*" OR movement*) AND ("inertial measurement unit" OR "IMU" OR "accelerometer" OR "inertial sensor" OR "wearable" OR "MEMS" or "magnetometer") AND ("Validity" OR "Accuracy" OR "Reliability" OR "Precision" OR "Varia*” OR "Repeatability" OR "Reproducibility" OR "Consistency" OR "noise”) AND ("jump*"). Additionally, the reference lists of the studies retrieved were manually searched to identify potentially eligible studies not captured by the electronic searches. Finally, an external expert was contacted to review the final list of references to determine whether there existed relevant studies that were not detected during the literature search. Possible errata were searched for each included study.

\section{Data extraction}

Data extraction was prepared in Microsoft Excel (Microsoft Corporation, Redmond, WA, USA) in ac- cordance with the Cochrane Consumers and Communication Review Group's data extraction template [29]. The Excel sheet was used to assess inclusion requirements and subsequently test for all selected studies. The process was independently conducted by 2 authors (FMC and MRG). Any disagreement regarding study eligibility was resolved in a discussion. Full text articles that were excluded were recorded with the reasons for exclusion. All the records were stored in the Excel sheet.

\section{Data items}

The following information was extracted from the included original articles: (i) validity measure (e.g., typical error, mean absolute error); and (ii) reliability measure (e.g., intraclass correlation coefficient [ICC] and/or typical error of measurement (\%) and/or coefficient of variation [CV] (\%) and/or standard error of measurement). Additionally, the following data items were extracted: (i) type of study design, number of participants (n), age group (youth, adult, or both), sex (men, women, or both), training level (untrained, trained); (ii) characteristics of the IMU and comparator (force plates); (iii) characteristics of the experimental approach to the problem, procedures, and settings of each study.

\section{Methodological assessment}

The methodological assessment process was performed by 2 authors (JPO and MRG) by using an adapted version of the STROBE assessment criteria for cross-sectional studies [30]. Each article was assessed on the basis of 10 specific criteria (Table 2). Any disagreement was discussed and solved by a consensus decision. Each item was evaluated with numerical characterization $(1=$ completed or $2=$ non-completed $)$. As suggested by O'Reilly et al. [30], each study rating was qualitatively interpreted as follows: a study had a high risk of bias or low quality with less than a total of 7 points, while studies with a greater total score were considered as low risk of bias or high quality.

\section{Ethical approval}

The conducted research is not related to either human or animal use. 


\section{HUMAN MOVEMENT}

F.M. Clemente et al., Inertial measurement units for jump height estimations

\section{Results}

\section{Study identification and selection}

The searching of the databases identified a total of 596 titles (Cochrane Library: 26; EBSCO: 204; PubMed: 122; SPORTDiscus: 142; and Web of Science: 102). These studies were then exported to reference manager software (EndNote ${ }^{\mathrm{TM}} \mathrm{X} 9$, Clarivate Analytics, Philadelphia, PA, USA). Duplicates (323 references) were subsequently removed, either automatically or manually. The remaining 273 articles were screened for their relevance on the basis of titles and abstracts, which resulted in the removal of further 213 studies. After the screening procedure, 60 articles were selected for in-depth reading and analysis. After reading full texts, further 30 studies were excluded as not meeting the eligibility criteria (Figure 1). Finally, a total of 30 articles were included in this review.

\section{Methodological quality}

The overall methodological quality of the crosssectional studies can be found in Table 2. A total of 24 $(80 \%)$ of the 30 included articles presented high methodological quality, while the remaining 6 studies (20\%) presented low methodological quality.

\section{Characteristics of individual studies}

The characteristics of the included studies can be found in Table 3. From the 30 included articles, 28 tested IMU validity [11, 17-19, 25, 26, 31-52], while 16 tested
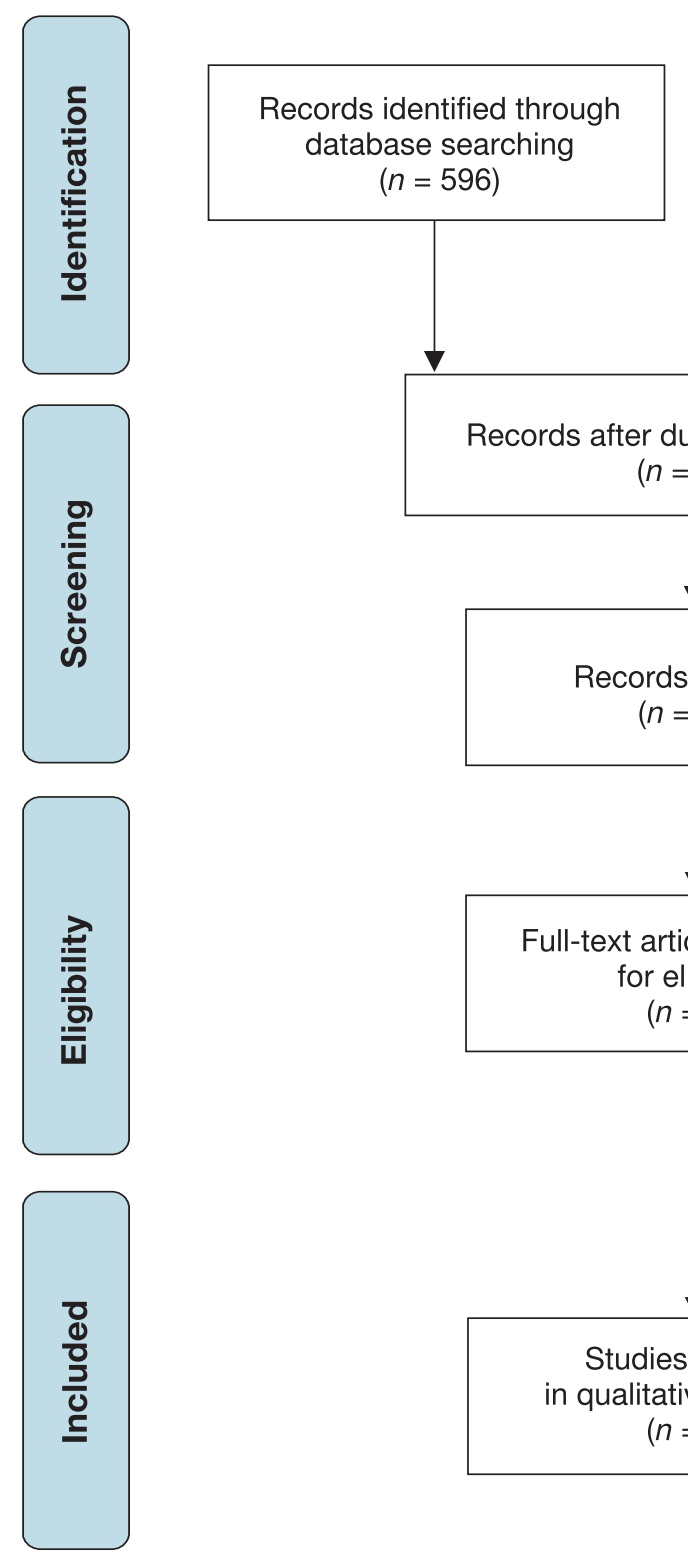
Additional records identified through other sources $(n=0)$

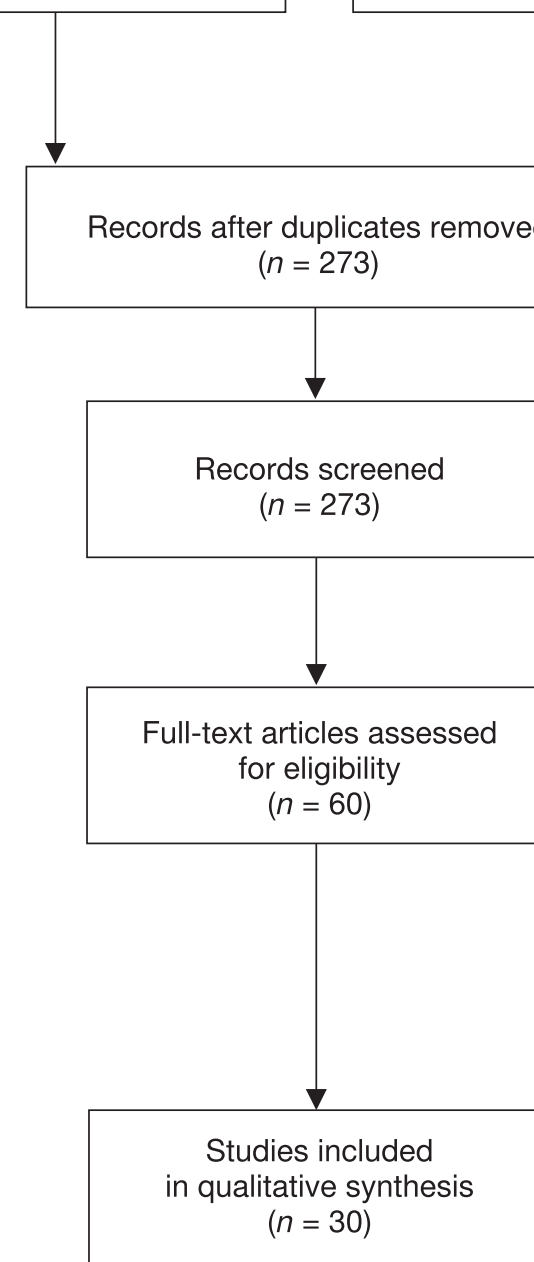

Records excluded $(n=213)$ 
Table 2. Methodological assessment of the included studies

\begin{tabular}{|c|c|c|c|c|c|c|c|c|c|c|c|}
\hline Reference & 1 & 2 & 3 & 4 & 5 & 6 & 7 & 8 & 9 & 10 & Quality \\
\hline Benson et al. [18] & 1 & 1 & 1 & 1 & 1 & 1 & 1 & 1 & 1 & 1 & High \\
\hline Borges et al. [49] & 1 & 1 & 1 & 1 & 1 & 1 & 1 & 1 & 1 & 0 & High \\
\hline Brooks et al. [50] & 1 & 1 & 1 & 1 & 1 & 1 & 1 & 0 & 1 & 0 & High \\
\hline Casartelli et al. [51] & 1 & 0 & 1 & 1 & 1 & 1 & 1 & 1 & 1 & 1 & High \\
\hline Charlton et al. [19] & 1 & 0 & 1 & 1 & 1 & 1 & 1 & 1 & 0 & 1 & High \\
\hline Choukou et al. [52] & 1 & 0 & 1 & 1 & 1 & 1 & 1 & 0 & 0 & 1 & Low \\
\hline Dowling et al. [31] & 1 & 0 & 1 & 1 & 1 & 1 & 1 & 0 & 0 & 1 & Low \\
\hline Gageler et al. [32] & 1 & 0 & 1 & 1 & 1 & 1 & 1 & 0 & 0 & 0 & Low \\
\hline Grainger et al. [33] & 1 & 1 & 0 & 1 & 1 & 1 & 1 & 1 & 1 & 0 & High \\
\hline Heredia-Jimenez and Orantes-Gonzalez [34] & 1 & 1 & 1 & 1 & 1 & 1 & 1 & 1 & 1 & 1 & High \\
\hline Hojka et al. [35] & 1 & 1 & 1 & 1 & 1 & 1 & 1 & 0 & 1 & 1 & High \\
\hline Lesinski et al. [36] & 1 & 1 & 1 & 1 & 1 & 1 & 1 & 1 & 0 & 1 & High \\
\hline MacDonald et al. [11] & 1 & 0 & 1 & 1 & 1 & 1 & 0 & 1 & 0 & 0 & Low \\
\hline Magnúsdóttir et al. [37] & 1 & 1 & 1 & 1 & 1 & 1 & 1 & 0 & 0 & 1 & High \\
\hline Mahmoud et al. [38] & 1 & 0 & 0 & 1 & 1 & 1 & 1 & 0 & 0 & 0 & Low \\
\hline Martínez-Martí et al. [39] & 1 & 1 & 1 & 1 & 1 & 1 & 1 & 1 & 1 & 1 & High \\
\hline Montoye and Mitrzyk [40] & 1 & 0 & 1 & 1 & 1 & 1 & 1 & 1 & 1 & 1 & High \\
\hline Nickerson et al. [53] & 1 & 0 & 1 & 1 & 1 & 1 & 1 & 1 & 1 & 1 & High \\
\hline Nuzzo et al. [54] & 1 & 0 & 1 & 1 & 1 & 1 & 1 & 0 & 1 & 1 & High \\
\hline Picerno et al. [25] & 1 & 0 & 1 & 1 & 1 & 1 & 1 & 1 & 0 & 1 & High \\
\hline Rago et al. [41] & 1 & 0 & 1 & 1 & 1 & 1 & 1 & 1 & 0 & 1 & High \\
\hline Rantalainen et al. [26] & 1 & 1 & 1 & 1 & 1 & 1 & 1 & 1 & 1 & 0 & High \\
\hline Rantalainen et al. [42] & 1 & 1 & 1 & 1 & 1 & 1 & 1 & 1 & 1 & 1 & High \\
\hline Skazalski et al. [17] & 1 & 0 & 0 & 1 & 1 & 1 & 0 & 1 & 0 & 0 & Low \\
\hline Spangler et al. [43] & 1 & 0 & 1 & 1 & 1 & 1 & 1 & 0 & 1 & 1 & High \\
\hline Stanton et al. [44] & 1 & 0 & 1 & 1 & 1 & 1 & 1 & 1 & 1 & 0 & High \\
\hline Toft Nielsen et al. [45] & 1 & 0 & 0 & 1 & 1 & 1 & 1 & 1 & 1 & 1 & High \\
\hline Wang et al. [48] & 1 & 1 & 1 & 1 & 1 & 1 & 1 & 1 & 1 & 1 & High \\
\hline Watkins et al. [46] & 1 & 1 & 1 & 1 & 1 & 1 & 1 & 1 & 0 & 1 & High \\
\hline Zihajehzadeh et al. [47] & 1 & 0 & 1 & 1 & 1 & 1 & 1 & 1 & 1 & 0 & High \\
\hline
\end{tabular}

Provide in the abstract an informative and balanced summary of what was done and what was found (item 1). State specific objectives, including any prespecified hypotheses (item 2). Give the eligibility criteria, and the sources and methods of selection of participants (item 3). For each variable of interest, give sources of data and details of methods of assessment (measurement). Describe comparability of assessment methods if there is more than one group (item 4). Explain how quantitative variables were handled in the analyses. If applicable, describe which groupings were chosen and why (item 5). Give characteristics of study participants (item 6). Summarize key results with reference to study objectives (item 7). Discuss limitations of the study, considering sources of potential bias or imprecision. Discuss both direction and magnitude of any potential bias (item 8). Give a cautious overall interpretation of results considering objectives, limitations, multiplicity of analyses, results from similar studies, and other relevant evidence (item 9). Give the source of funding and the role of the funders for the present study and, if applicable, for the original study on which the present article is based (item 10).

IMU reliability [11, 17-19, 25, 26, 31, 41, 43, 46, 48, 50-54]. Ten of the included studies compared the IMU device with force platforms [17, 26, 34-36, 44-46, 50, 52]. Twelve studies compared with video motion capture systems [11, 17-19, 25, 31-33, 37-39, 43]. Three studies compared with Vertec [40, 49, 54]. Four studies compared with photoelectric systems [34, 36, 39, 51]. One study compared with VERT [53]. Among the included studies, 12 used CMJ [11, 33-36, 39, 50-55].
Six studies used SJ [36, 39, 46, 51, 52, 55]. Two studies used the Abalakov jump [37, 39]. Also, 4 studies used other sport-specific jumps, such as the block jump in volleyball, among others [40, 44, 46, 47].

Overall, 16 different IMUs were tested. A total of 8 studies tested the VERT [11, 17-19, 38, 49, 50, 53]. Six studies tested the Myotest [35, 41, 44, 51, 52, 55]. Two studies tested the FreePower [25, 34]. Two studies tested the MinimaxX GPS [26, 43]. The GPSport, Xsens 


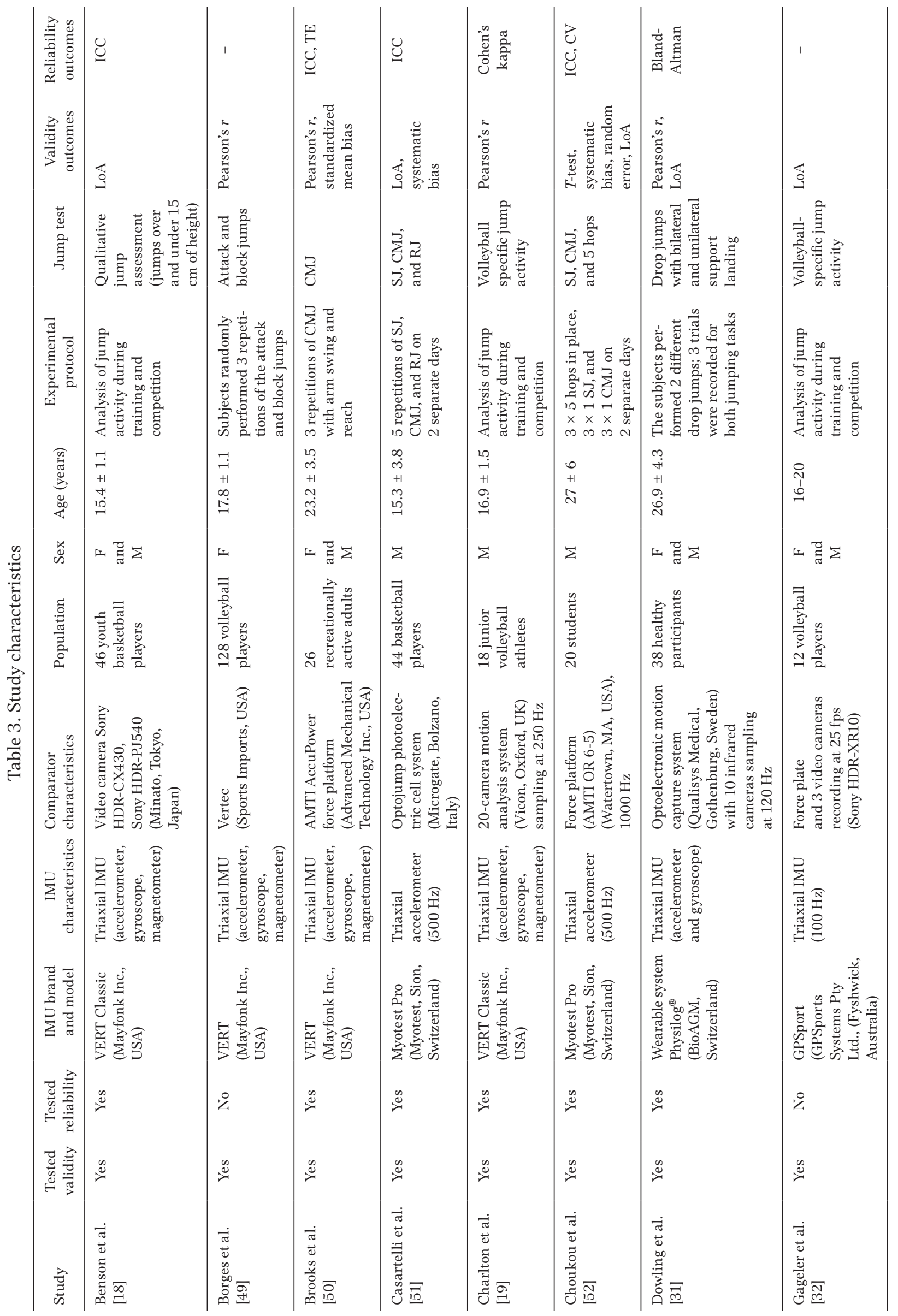




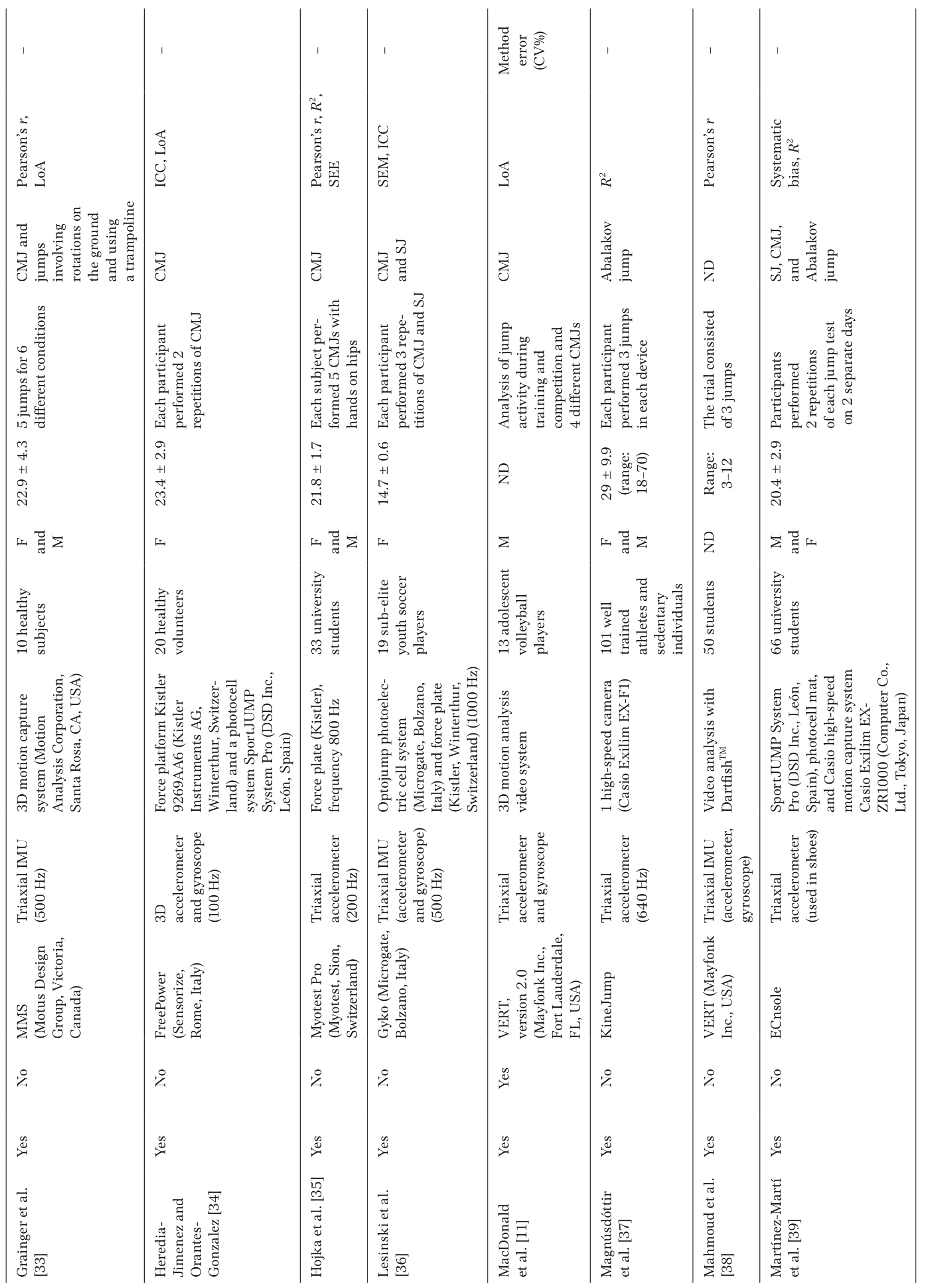




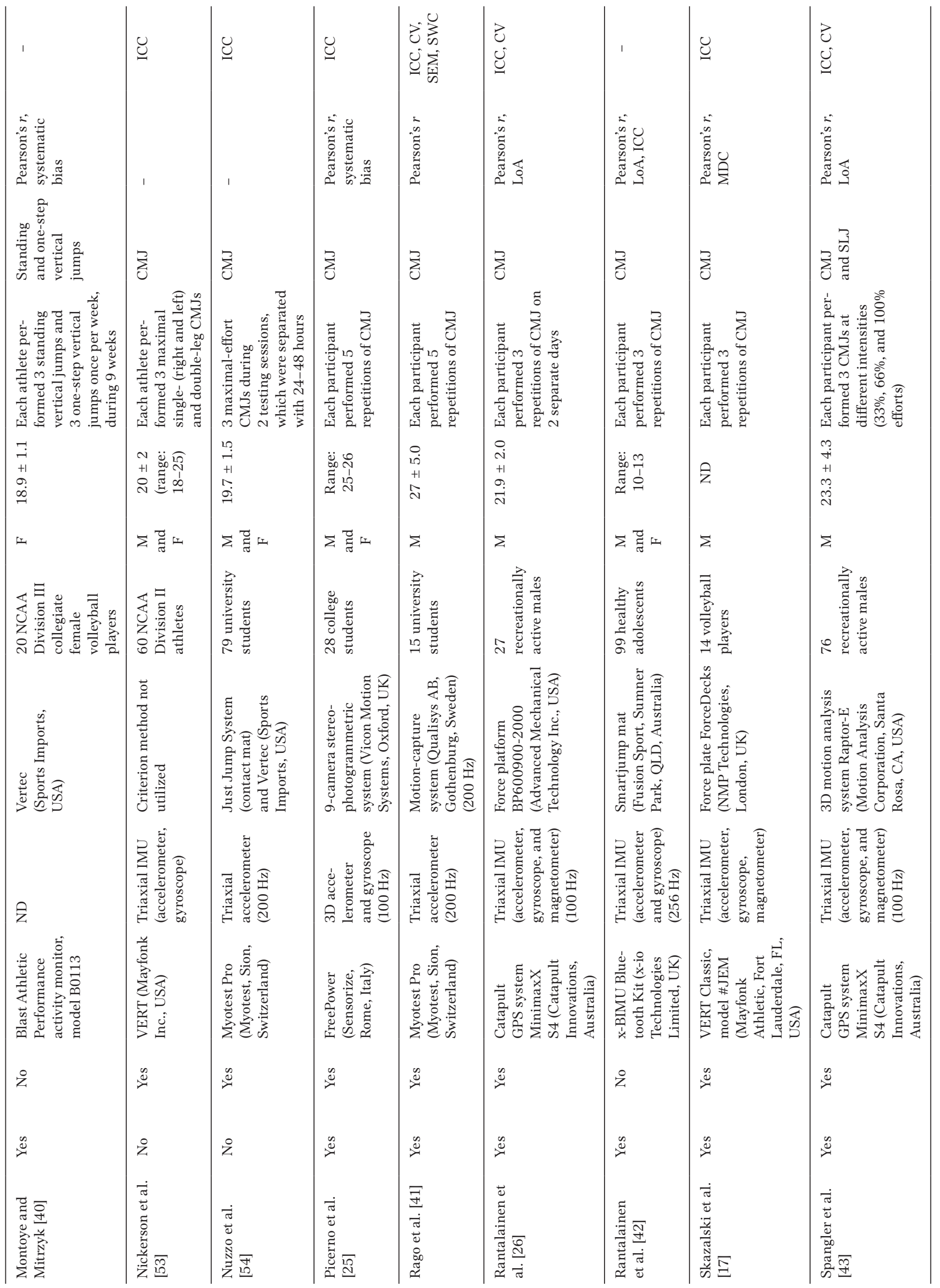




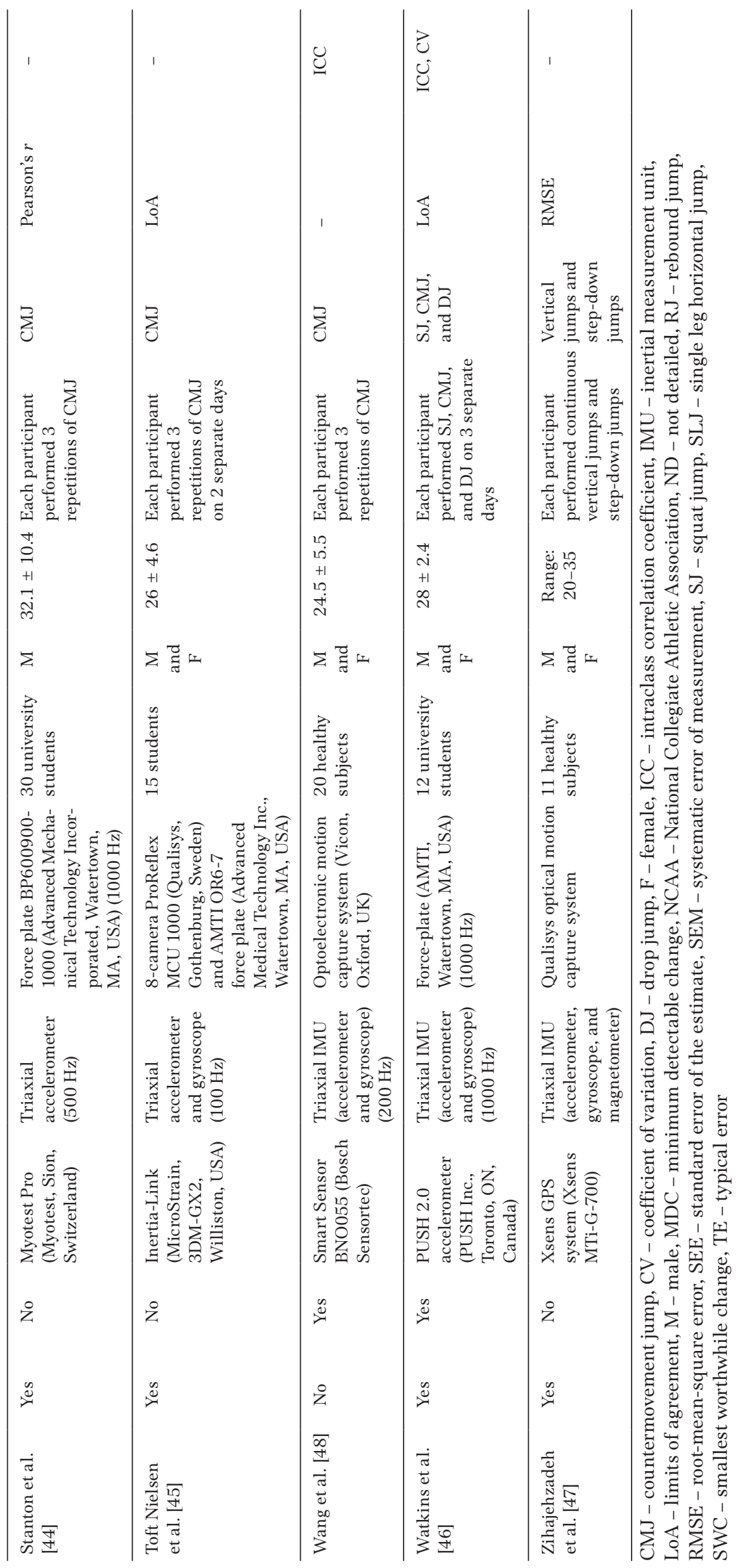


GPS, PUSH, KineJump, Gyko, ECnsole, Physilog ${ }^{\circledR}$, MMS, $x$-BIMU, Inertia-Link, Blast Athletic, and Smart Sensor IMUs were tested only once [31-33, 36, 37, 39, $40,42,45-48]$.

Results of individual studies: validity of IMU for estimation of jump height

Information on the validity levels obtained in the included studies can be found in Tables 4-7. For the VERT device, the Pearson correlation coefficient values of validity were between $r=0.75$ and 0.99 [11, 19, 38, $49,50]$, and limits of agreement values were between -12.6 and $33.8 \mathrm{~cm}[11,18,19]$. For the Myotest device, the Pearson values were between $r=0.66$ and 0.93 [35, 41, 44], and limits of agreement values were between -13.4 and $53.4 \mathrm{~cm}[44,51,52]$. For the FreePower device, a Pearson value of $r=0.87$ [25] and an ICC value of 0.93 [34] were presented, while limits of agreement were between -0.07 and $0.06 \mathrm{~m}$ [34]. For the MinimaxX GPS, the Pearson values were between $r=0.722$ and 0.969 [26, 43], and limits of agreement values between -14.41 and $19.2 \mathrm{~cm}$ [26, 43]. For the IMU devices that were tested only once for validity, the Pearson values were between $r=0.67$ and 0.99 [31, $33,37,40,48$, and limits of agreement values between -15.6 and $19.2 \mathrm{~cm}[31-33,42,45,46]$.

Results of individual studies: reliability of IMU for estimation of jump height

Information on the reliability levels obtained in the included studies can be found in Tables 8-10. For the VERT device, the ICC values of reliability were between 0.786 and 0.997 [17, 18, 50, 53], and CV\% values reached $4.4 \%$ [11]. For the Myotest device, the ICC values were between 0.56 and 0.97 [41, 51, 52, 54], and CV\% values ranged 3.62-13.23\% [41, 51, 52, 54]. For the Mini$\operatorname{maxX}$ GPS, the ICC values were between 0.720 and 0.959 [26, 43], and CV\% values ranged $6.7-17 \%[26,43]$. For the IMU devices that were tested only once for reliability, the ICC values were between 0.83 and 0.986 [25, 46, 48], and CV\% values ranged 3.7-6.0\% [46].

\section{Discussion}

This systematic review aimed (1) to identify and summarize studies that have examined the validity of IMU devices for measuring jump height and (2) to identify and summarize studies that have examined the reliability IMU devices for measuring jump height. Although the IMU devices presented overall promis-

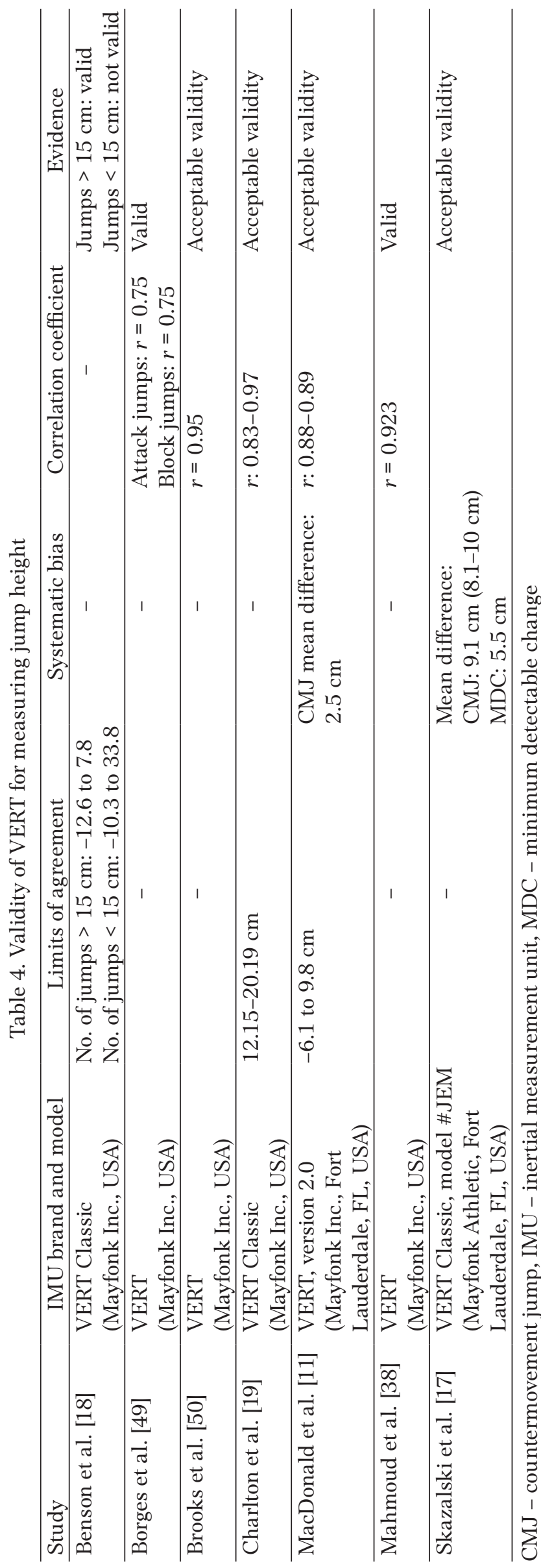




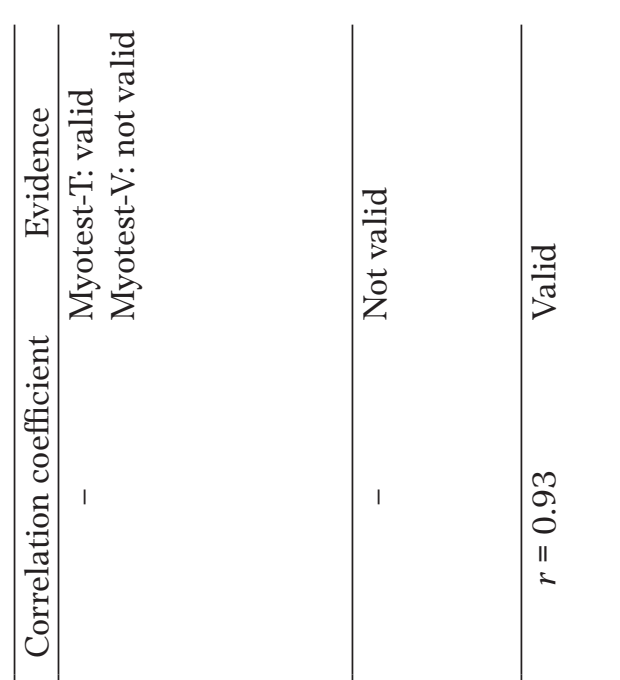

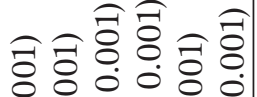

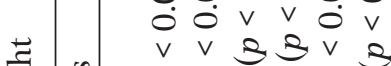

:

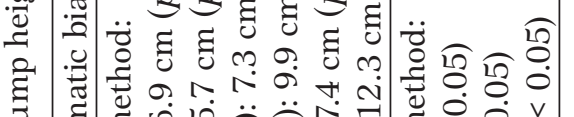

至

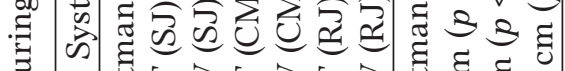

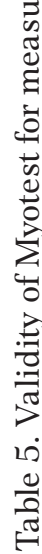

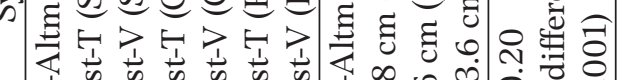

它

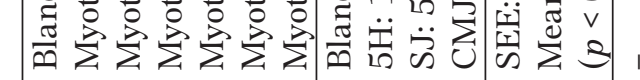

范

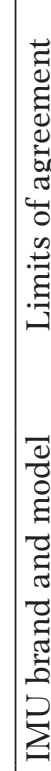

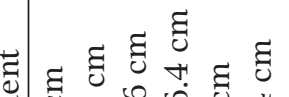

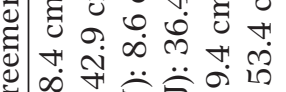

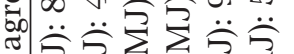

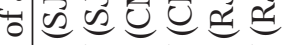

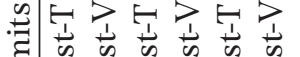

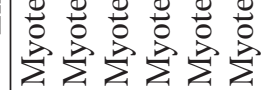

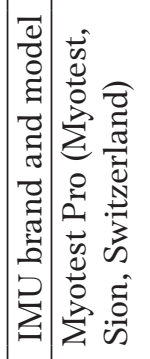

竞

$\ddot{\bar{\Xi}}$

意

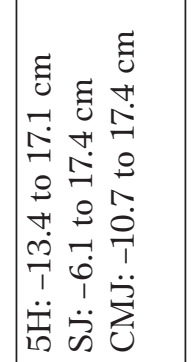

言离完

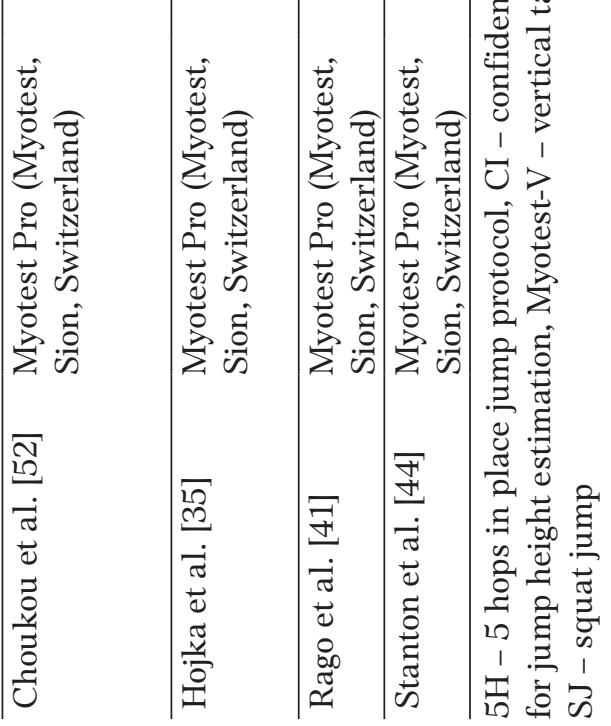

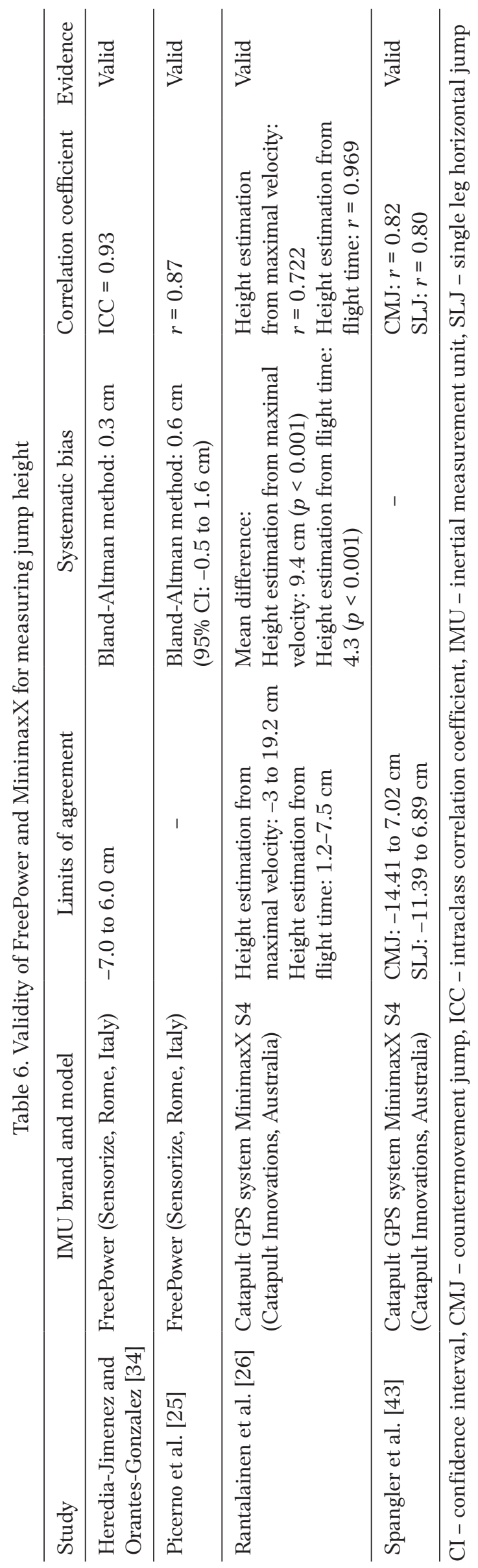




\section{HUMAN MOVEMENT}

F.M. Clemente et al., Inertial measurement units for jump height estimations

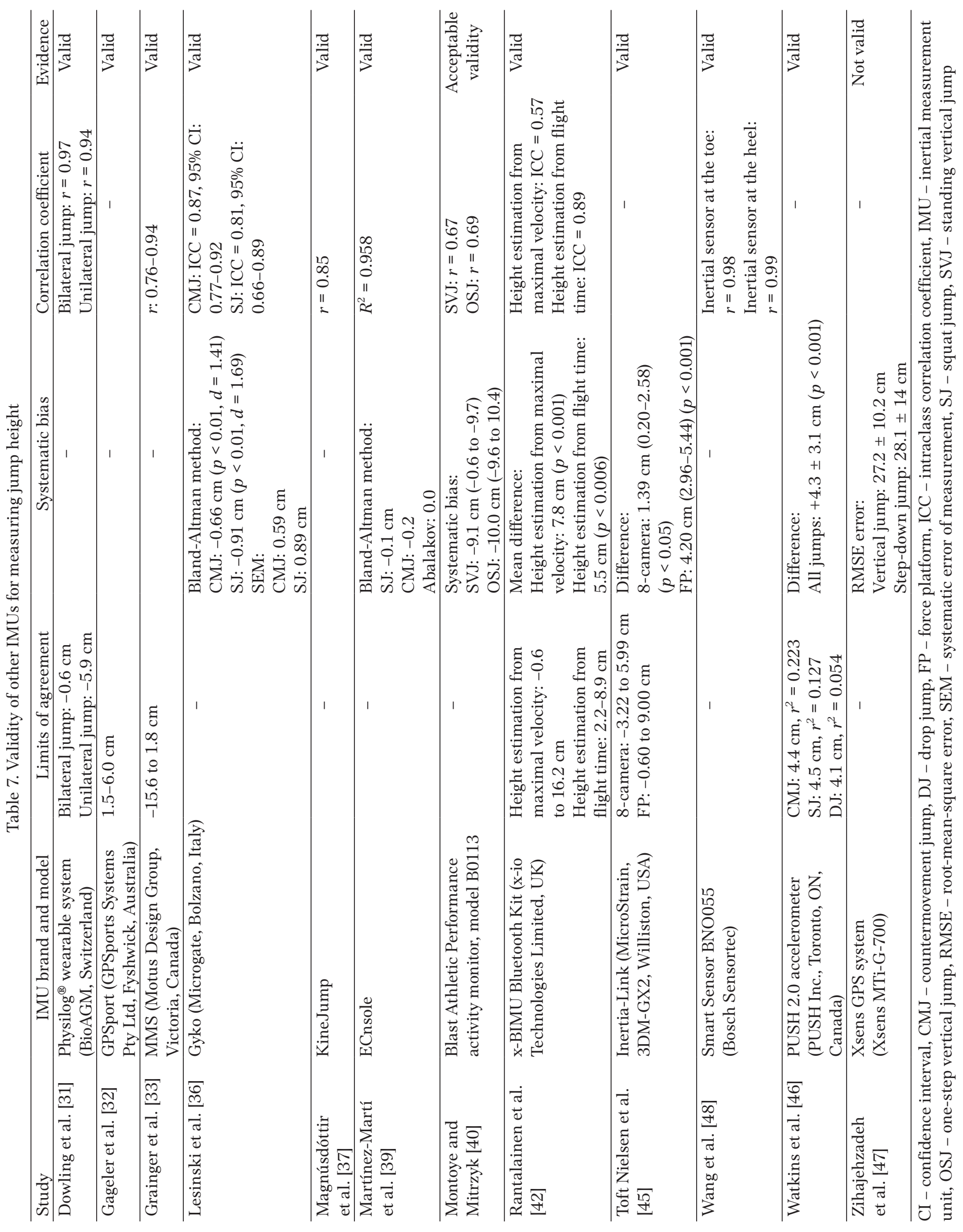



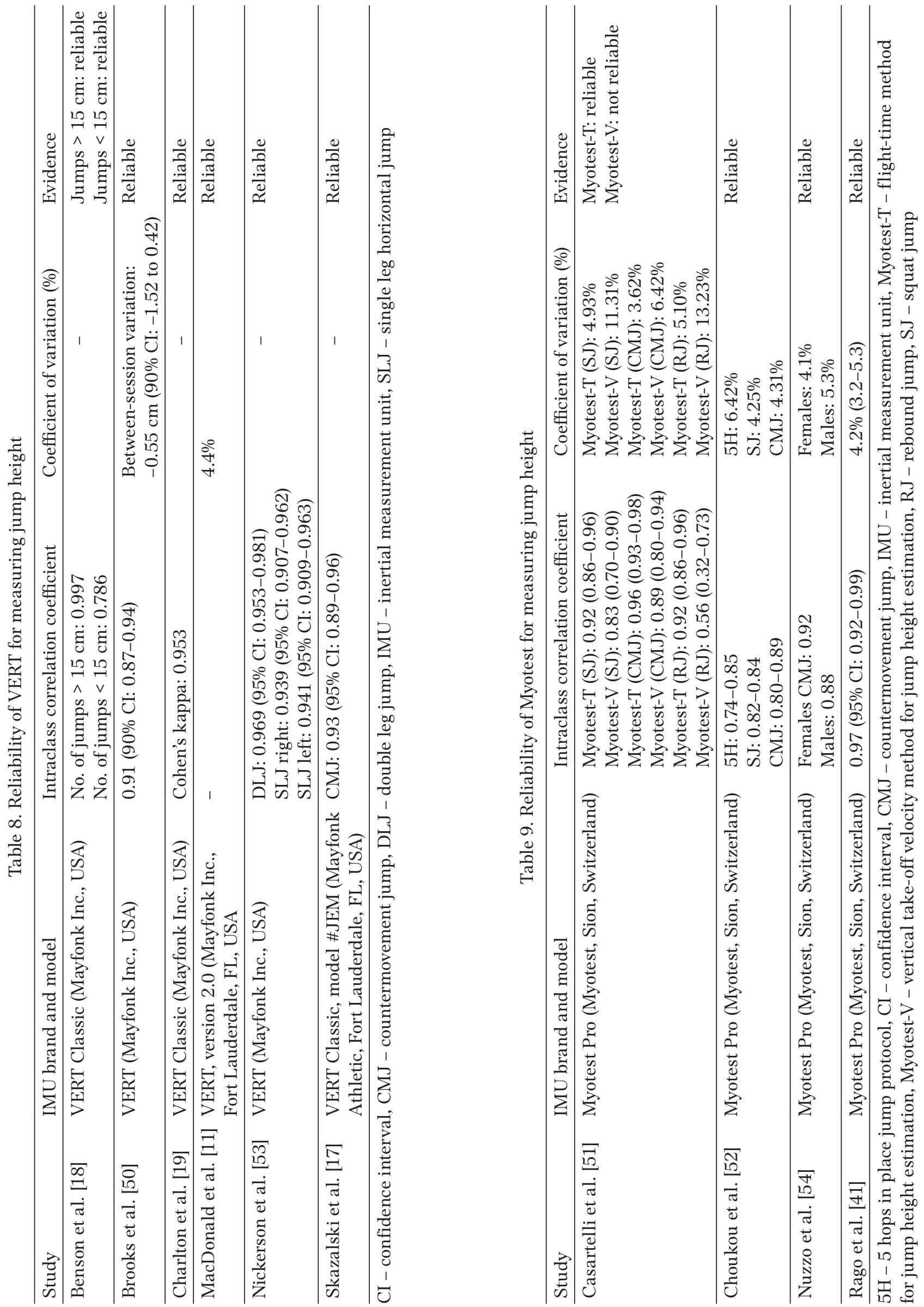
ing results, some of them may not be the best choice to use as a 'stand-alone' jump height assessment device, given the wide limits of agreement and coefficients of variation revealed. Also, only 4 of the 16 IMUs included (VERT, Myotest, FreePower, and MinimaxX) were tested more than once $[11,17-19,25,26,34,35,38$, $41,43,44,49-52]$. All other IMUs involved in the present systematic review (GPSport, Xsens GPS, PUSH, KineJump, Gyko, ECnsole, Physilog ${ }^{\circledR}$, MMS, x-BIMU, Inertia-Link, Blast Athletic, and Smart Sensor) were tested only once [31-33, 36, 37, 39, 40, 42, 45-48].

\section{Validity of IMUs for estimation of jump height}

From the 16 IMUs included in the present systematic review, the most tested for validity was the VERT IMU device [11, 17-19, 38, 49, 50]. Overall, the authors of the studies that tested VERT validity demonstrated that it was a valid device to measure jump height. However, it must be addressed that some authors tested the VERT device for measuring jump activity during training and competition $[18,19]$, while others tested the device using $\mathrm{CMJ}, \mathrm{SJ}$, and other sport-specific jump tests [11, 17, 38, 49, 50].

The 2 studies that analysed the validity of the VERT device to measure jump activity during training and competition revealed only acceptable validity for evaluating vertical displacement and jump count $[18,19]$. Although VERT showed good agreement with the goldstandard instrument (video camera), it turned out unable to detect jumps below $15 \mathrm{~cm}$ during basketball practice and competition [18]. Another study, conducted among 18 junior volleyball players, demonstrated acceptable validity for the VERT device when compared with a 20 -camera motion analysis system for measuring jump height during volleyball training and competition [19]. Although VERT exhibited good-to-excellent correlations with the reference method, the limits of agreement for volleyball-specific jumps, such as the set jump and the spike jump, seem to be wide [19].

With regard to VERT as applied to assess jump height during jump testing protocols, MacDonald et al. [11] observed strong correlations between VERT and a 3D motion analysis video system ( $r: 0.88-0.89)$, and narrower limits of agreement $(-6.1$ to $9.8 \mathrm{~cm})$ when using a $\mathrm{CMJ}$ test protocol in relation to that found by Charlton et al. [19]. However, the same authors [11] also revealed that VERT underestimated maximal jump height by $2.5 \mathrm{~cm}$ when compared with the reference method. Also, Brooks et al. [50] showed a strong correlation $(r=0.95)$ between VERT and a force platform. However, the authors affirmed that VERT was not ac- 
curate enough to detect small changes in performance given its associated moderate standardized typical errors found. This is of particular importance as for elite sport environments, devices capable of detecting small changes in performance are prioritized.

VERT seems to be a good and less time-consuming alternative to quantify jump load during training and competition of indoor team sports, such as basketball and volleyball. However, given that only 2 studies tested VERT validity and these referred to youth non-elite populations, more studies are needed to make generalizations regarding this IMU validity to quantify jump activity in practice, especially in elite adult populations. Using the VERT device to track changes in jump performance over time in elite sport populations is not the best option in relation to other, more affordable and sensible units, such as the My Jump 2 application, for example [56]. Also, the studies that tested VERT validity used Pearson correlation coefficients to set the level of the device accuracy. This statistical method may not be the most appropriate to analyse the agreement of a device with a gold-standard measure; the Bland-Altman method is preferred to analyse the limits of agreement and their related confidence intervals [57, 58].

Considering the Myotest device, contradictory findings were observed between the analysed studies. While Hojka et al. [35] showed a strong relationship between Myotest and a force plate $(r=0.93)$ for measuring CMJ performance, Stanton et al. [44] revealed a lower correlation between the same device and a force plate. However, the study by Hojka et al. [35] presented greater jump height differences between Myotest and the force plate than Stanton et al. [44]. Furthermore, Choukou et al. [52] reported wide limits of agreement for the CMJ height measure using Myotest when compared with a force platform. The same study demonstrated a systematic bias of approximately $4 \mathrm{~cm}$ and $6 \mathrm{~cm}$ for SJ and CMJ, respectively, between the devices. For those reasons, the authors suggested that Myotest was not valid for assessing CMJ and SJ height [52]. Another study also revealed a similar bias of approximately $7 \mathrm{~cm}$ for $\mathrm{CMJ}$ performance between Myotest and a photoelectric system [51]. Although the abovementioned study showed a bias similar to that presented by Choukou et al. [52], the authors claimed the validity of the Myotest device to measure jump height given the narrower limits of agreement found [51].

These discrepancies between studies are somewhat expected as methodologic differences were observed between them. In fact, while the studies were in general conducted among university students [35, 41, 44, 52], only one study was performed in athletes [51] to test the Myotest device validity. Also, different jump testing protocols were used. From a practical perspective and given the importance of such devices for measuring performance changes in athlete populations, more research is required to test the validity of Myotest in elite sport contexts.

Only 2 studies investigated the validity of the FreePower device [25, 34], and other 2 studies were devoted to the MinimaxX GPS system [26, 43]. The authors of these studies claimed the validity of both FreePower and MinimaxX GPS IMUs for measuring jump height by using CMJ testing protocols [25, 26, 34, 43]. In fact, Picerno et al. [25] found significantly high correlations between the FreePower device and a motion capture system, used as a reference method. The same authors also revealed low bias $(0.6 \mathrm{~cm})$ between the devices, confirming FreePower validation [25]. Similarly, the other study demonstrated low bias $(0.3 \mathrm{~cm})$ between FreePower and a force platform when using the flighttime method to estimate jump height from the CMJ test, as well as relatively narrow limits of agreement between the IMU and the force platform [34]. For the MinimaxX GPS system, promising results were found, given the usefulness of such a device in outfield team sport settings. The 2 studies that tested validity reported strong correlations between the IMU and both 3 D motion analysis system and a force platform [26, 43]. However, the highest correlation was between the IMU and the force platform [26]. Despite the strong correlations between MinimaxX and the reference methods from both studies, using the force platform as the gold-standard method produced narrower limits of agreement than comparing the IMU with a 3D motion analysis system [26, 43].

As mentioned earlier, all other IMU devices were tested only once for validity [31-33, 36, 37, 39, 40, 42, 45-48]. Overall, the studies claimed the validity of 12 different IMUs. Only the Xsens GPS system was not valid for measuring jump height [47]. In fact, that study revealed large systematic errors of approximately $28 \mathrm{~cm}$ between the GPS system and an optical system as a reference method [47]. In contrast, a study which investigated the validity of another GPS system (GPSport) to measure jump height demonstrated its validity [32]. This latter study compared the IMU device with a force plate and revealed acceptable limits of agreement. The authors suggested the IMU validity given that using this method of measuring jump height was beneficial in elite sport settings because of the shorter time needed to conduct such assessments when compared with other, traditional methods, e.g., video 
F.M. Clemente et al., Inertial measurement units for jump height estimations

analysis or manual entries (frame-by-frame) in jump applications [32, 56]. The GPSport IMU produced similar limits of agreement to those from the MinimaxX GPS system. This is potentially promising as in elite sports, mainly in outfield team sports, teams apply GPS systems to quantify external loads during training and competition, as well as a complement to the return-to-competition processes [59, 60]. Given the current time constraints of elite sports, such as soccer, using this type of device to measure the physical fitness status of players can reduce this issue difficulties and promote a more responsive action from practitioners to better inform coaches regarding their players' status [61].

\section{Reliability of IMUs for estimation}

of jump height

From the 30 studies included in the present systematic review, only 16 tested the reliability of the different IMUs, with all studies claiming the reliability of all devices [11, 17-19, 25, 26, 31, 41, 43, 46, 48, 50-54]. The VERT device was the most tested for reliability [11, 17-19, 50, 53]. The studies that analysed jump activity during training and competition revealed excellent reliability when compared with a video analysis system [18] and a force platform [50]. Similarly, VERT was observed to be reproducible when used to measure jump height in testing settings, with ICC values ranging between 0.93 and 0.96 for both double and single leg vertical jumps $[17,53]$. Although the ICC values of the overall studies pointed at excellent reliability of the VERT IMU device, only one study reported CV\% values (4\%) [11]. Given that test-retest correlations are sensitive to the heterogeneity of a given sample, the use of CV\% and changes in the mean are preferred to analyse the reliability of devices that have the potential to be used to track changes in athletes' performance [62].

The reliability of the Myotest IMU was investigated in 4 studies [41, 51, 52, 54]. The 4 studies revealed that Myotest was a reliable instrument to measure jump height performance. In fact, CV\% values between 3.6\% and 5.3\% were found for SJ and CMJ heights, with ICC values between 0.80 and 0.97 . However, most of these studies were conducted among university students, while only one was performed in athletes [51]. Also, while some studies used a jump test protocol consisting in 3 repetitions [52, 54], others applied 5 repetitions $[41,51]$ of each jump test, which can be a determinant factor for producing smaller or larger $\mathrm{CV} \%$ [62]. Furthermore, it seems that the Myotest device is more reproducible for assessing jump height, mainly in more controlled jumps, such as SJ and CMJ, as the 5 hop in place jump test (less movement control) has greater within-subject variation [52].

Two studies were conducted to test the reliability of the MinimaxX GPS system [26, 43]. Although both claimed the reliability of the MinimaxX, higher CV\% values than those found for the VERT and Myotest IMUs were reported. In fact, both studies [26, 43] demonstrated CV\% between $6.7 \%$ and $7.3 \%$ for jump height. Notwithstanding those higher values of variation, the MinimaxX presented excellent ICC values for test-retest for all jump heights. Despite that, the VERT and Myotest seem to be more reliable to measure jump performance than the MinimaxX GPS system. However, the 2 studies that examined the MinimaxX reliability were conducted among recreational individuals, who usually do not use this type of equipment to measure changes in jump performance over time. Given the fact that GPS systems can present much more utility in elite team sport contexts [60], future studies analysing the reliability of such devices should be performed in elite athlete populations to allow better generalizations. This would be of great interest for both coaches and practitioners, as time constraints would be softened if such devices were proved reliable in this population.

For the Physilog ${ }^{\circledR}$, FreePower, Smart Sensor, and PUSH IMUs, only one study was conducted for each device to test their reliability, and all claimed the reliability [25, 31, 46, 48]. However, from these 4 IMUs, only the PUSH presented more interesting results [46]. In fact, only Watkins et al. [46] reported CV\% values along with the ICC values. These authors observed CV\% values similar to those found in the VERT, Myotest, and MinimaxX reliability studies, ranging from 3.7\% to $6.0 \%$, depending on the type of jump. Indeed, the most controlled jumps (e.g., SJ and CMJ) had lower CV\% values compared with a drop jump (less movement control), as determined by Choukou et al. [52], who tested the reliability of the Myotest IMU device.

Although the results presented are interesting, future studies to investigate the reliability of IMUs should be designed for long-term use in order to minimize the effects of variables that may arise as a result of biological differences, and conducted in elite sport contexts. Also, it is thought that manufacturer-induced malfunctions may occur in the software data flow and that disruptions in this type of data may affect IMU reliability outcomes. 
Study limitations, future research, and practical implications

The limitations of this research include the fact that the validity and reliability results presented in this systematic review represented the use of IMUs in controlled environments, as all of the involved studies were performed in laboratory settings. Therefore, they cannot be considered directly representative of IMU performance during real-sport scenarios. Considering variability, the included studies showed that IMUs were reliable tools to measure jump height performance, mainly in recreationally active individuals, as studies conducted in elite sport populations are lacking. The studies discussed in this systematic review generally consist of short-term research designs. Since the use of IMUs in sports contexts is to assess human performance, and given the fact that humans present great biological within- and between-subject differences, it should be noted that long-term changes in performance may affect the validity and reliability of IMUs. To the best of the authors' knowledge, no studies have examined the validity and reliability of long-term IMU application. Therefore, future studies should investigate the validity and reliability of the available IMUs in longterm settings. Also, more consistent and homogeneous jumping test protocols and the use of a reference method that is most applied in sports contexts, such as the increasingly available force platforms, must be considered in future studies.

IMU devices can be used to assess jump height in non-elite and recreationally active individuals. However, if the aim is to utilize them in elite athletic populations, such devices may not be sensible enough to detect small changes in jump height performance. This is of paramount importance, as in elite settings, the instruments used to track variations in athletes' performance need to be capable of detecting even the smallest changes. Despite that, the VERT, Myotest, MinimaxX GPS, and PUSH devices presented the most promising results. Still, further investigations are needed to confirm their validity and reliability in elite sport populations.

\section{Conclusions}

The present systematic review summarized the evidence on the validity and reliability of IMUs for measuring jump height. Although the IMUs were considered valid in general, the Xsens GPS system was not valid. Despite the VERT and Myotest devices were considered valid, they seem not to be accurate enough to detect small changes in performance. From the 16 devices, only 7 were tested for reliability. All were considered reliable but the VERT, Myotest, MinimaxX GPS, and PUSH IMUs presented lower variation in jump height assessments. From the 16 devices, 15 can be used with acceptable accuracy and only 7 with acceptable precision. However, elite sports coaches and practitioners must interpret such findings with caution, as generalizations cannot be made for elite populations.

\section{Disclosure statement}

No author has any financial interest or received any financial benefit from this research.

\section{Conflict of interest}

The authors state no conflict of interest.

\section{Funding}

This work is funded by Fundação para a Ciência e Tecnologia/Ministério da Ciência, Tecnologia e Ensino Superior through national funds and, when applicable, co-funded by EU funds under the project UIDB/50008/2020.

\section{References}

1. Eagles AN, Sayers MGL, Bousson M, Lovell DI. Current methodologies and implications of phase identification of the vertical jump: a systematic review and meta-analysis. Sports Med. 2015;45(9):1311-1323; doi: 10.1007/s40279-015-0350-7.

2. Watkins CM, Barillas SR, Wong MA, Archer DC, Dobbs IJ, Lockie RG, et al. Determination of vertical jump as a measure of neuromuscular readiness and fatigue. J Strength Cond Res. 2017;31(12):3305-3310; doi: 10.1519/JSC.0000000000002231.

3. Petrigna L, Karsten B, Marcolin G, Paoli A, D’Antona G, Palma A, et al. A review of countermovement and squat jump testing methods in the context of public health examination in adolescence: reliability and feasibility of current testing procedures. Front Physiol. 2019;10: 1384; doi: 10.3389/fphys.2019.01384.

4. Van Hooren B, Zolotarjova J. The difference between countermovement and squat jump performances: a review of underlying mechanisms with practical applications. J Strength Cond Res. 2017;31(7):2011-2020; doi: 10.1519/JSC.0000000000001913.

5. Balsalobre-Fernández C, Tejero-González CM, del Campo-Vecino J, Bavaresco N. The concurrent validity and reliability of a low-cost, high-speed camera-based method for measuring the flight time of vertical jumps. J Strength Cond Res. 2014;28(2):528-533; doi: 10.1519/ JSC.0b013e318299a52e.

6. Mathieu B, Peeters A, Piscione J, Lacome M. Usefulness of typical tests of short-duration maximal effort used 
F.M. Clemente et al., Inertial measurement units for jump height estimations

to assess players readiness to perform. Sport Perform Sci Rep. 2017;3(1):1-3.

7. Farias DL, Teixeira TG, Madrid B, Pinho D, Boullosa DA, Prestes J. Reliability of vertical jump performance evaluated with contact mat in elderly women. Clin Physiol Funct Imaging. 2013;33(4):288-292; doi: 10.1111/ cpf.12026.

8. Glatthorn JF, Gouge S, Nussbaumer S, Stauffacher S, Impellizzeri FM, Maffiuletti NA. Validity and reliability of Optojump photoelectric cells for estimating vertical jump height. J Strength Cond Res. 2011;25(2):556560; doi: 10.1519/JSC.0b013e3181ccb18d.

9. Moir GL. Three different methods of calculating vertical jump height from force platform data in men and women. Meas Phys Educ Exerc Sci. 2008;12(4):207-218; doi: 10.1080/10913670802349766.

10. Wadhi T, Rauch JT, Tamulevicius N, Andersen JC, De Souza EO. Validity and reliability of the GymAware linear position transducer for squat jump and counter-movement jump height. Sports. 2018;6(4):177; doi: 10.3390/sports6040177.

11. MacDonald K, Bahr R, Baltich J, Whittaker JL, Meeuwisse WH. Validation of an inertial measurement unit for the measurement of jump count and height. Phys Ther Sport. 2017;25:15-19; doi: 10.1016/j.ptsp.2016. 12.001.

12. McMaster DT, Tavares F, O’Donnell S, Driller M. Validity of vertical jump measurement systems. Meas Phys Educ Exerc Sci. 2021;25(2):95-100; doi: 10.1080/109 1367X.2020.1835664.

13. Nam Y, Kim Y, Lee J. Sleep monitoring based on a triaxial accelerometer and a pressure sensor. Sensors. 2016;16(5):750; doi: 10.3390/s16050750.

14. Clemente FM, Akyildiz Z, Pino-Ortega J, Rico-González M. Validity and reliability of the inertial measurement unit for barbell velocity assessments: a systematic review. Sensors. 2021;21(7):2511; doi: 10.3390/ s21072511.

15. Alanen AM, Räisänen AM, Benson LC, Pasanen K. The use of inertial measurement units for analyzing change of direction movement in sports: a scoping review. Int J Sports Sci Coach. 2021;16(6):1332-1353; doi: 10.1177/17479541211003064.

16. Camomilla V, Bergamini E, Fantozzi S, Vannozzi G. Trends supporting the in-field use of wearable inertial sensors for sport performance evaluation: a systematic review. Sensors. 2018;18(3):873; doi: 10.3390/s18030 873.

17. Skazalski C, Whiteley R, Hansen C, Bahr R. A valid and reliable method to measure jump-specific training and competition load in elite volleyball players. Scand J Med Sci Sports. 2018;28(5):1578-1585; doi: 10.1111/sms. 13052.

18. Benson LC, Tait TJ, Befus K, Choi J, Hillson C, Stilling $\mathrm{C}$, et al. Validation of a commercially available inertial measurement unit for recording jump load in youth basketball players. J Sports Sci. 2020;38(8):928936; doi: 10.1080/02640414.2020.1737360.

19. Charlton PC, Kenneally-Dabrowski C, Sheppard J, Spratford W. A simple method for quantifying jump loads in volleyball athletes. J Sci Med Sport. 2017; 20(3):241-245; doi: 10.1016/j.jsams.2016.07.007.

20. Rossi A, Pappalardo L, Cintia P, Pedreschi D, Iaia FM, Alberti G. The importance of GPS features to describe elite football training. SISMES Conference. 2016;12(Suppl. 1):27-28.

21. Ahmad N, Ghazilla RAR, Khairi NM, Kasi V. Reviews on various inertial measurement unit (IMU) sensor applications. Int J Signal Process Syst. 2013;1(2):256262; doi: 10.12720/ijsps.1.2.256-262.

22. Thompson SW, Rogerson D, Dorrell HF, Ruddock A, Barnes A. The reliability and validity of current technologies for measuring barbell velocity in the freeweight back squat and power clean. Sports. 2020;8(7): 94; doi: 10.3390/sports8070094.

23. Aughey RJ. Applications of GPS technologies to field sports. Int J Sports Physiol Perform. 2011;6(3):295-310; doi: 10.1123/ijspp.6.3.295.

24. O’Donoghue P, Papadimitriou K, Gourgoulis V, Haralambis K. Statistical methods in performance analysis: an example from international soccer. Int J Perform Anal Sport. 2012;12(1):144-155; doi: 10.1080/247486 68.2012.11868590.

25. Picerno P, Camomilla V, Capranica L. Countermovement jump performance assessment using a wearable 3D inertial measurement unit. J Sports Sci. 2011;29(2): 139-146; doi: 10.1080/02640414.2010.523089.

26. Rantalainen T, Gastin PB, Spangler R, Wundersitz D. Concurrent validity and reliability of torso-worn inertial measurement unit for jump power and height estimation. J Sports Sci. 2018;36(17):1937-1942; doi: 10.1080/02640414.2018.1426974.

27. McGrath J, Neville J, Stewart T, Cronin J. Upper body activity classification using an inertial measurement unit in court and field-based sports: a systematic review. Proc Inst Mech Eng P J Sport Eng Technol. 2021;235(2): 83-95; doi: 10.1177/1754337120959754.

28. Moher D, Liberati A, Tetzlaff J, Altman DG. Preferred Reporting Items for Systematic Reviews and Meta-Analyses: the PRISMA statement. PLoS Med. 2009;6(7): e1000097; doi: 10.1371/journal.pmed.1000097.

29. Cochrane Consumers and Communication. Data extraction template for included studies. 2016.

30. O’Reilly M, Caulfield B, Ward T, Johnston W, Doherty C. Wearable inertial sensor systems for lower limb exercise detection and evaluation: a systematic review. Sports Med. 2018;48(5):1221-1246; doi: 10.1007/s40279018-0878-4.

31. Dowling AV, Favre J, Andriacchi TP. A wearable system to assess risk for anterior cruciate ligament injury during jump landing: measurements of temporal events, jump height, and sagittal plane kinematics. J Biomech Eng. 2011;133(7):071008; doi: 10.1115/1.4004413. 
32. Gageler HW, Wearing S, James AD. Automatic jump detection method for athlete monitoring and performance in volleyball. Int J Perform Anal Sport. 2015; 15(1):284-296; doi: 10.1080/24748668.2015.11868793.

33. Grainger M, Weisberg A, Stergiou P, Katz L. Comparison of two methods in the estimation of vertical jump height. J Hum Sport Exerc. 2020;15(3):623-632; doi: 10.14198/jhse.2020.153.12.

34. Heredia-Jimenez J, Orantes-Gonzalez E. Comparison of three different measurement systems to assess the vertical jump height. Rev Bras Med Esporte. 2020;26(2): 143-146; doi: 10.1590/1517-869220202602185305.

35. Hojka V, Tufano JJ, Malý T, Št’astný P, Jebavý R, Feher J, et al. Concurrent validity of Myotest for assessing explosive strength indicators in countermovement jump. Acta Gymnica. 2018;48(3):95-102; doi: 10.5507/ag. 2018.013.

36. Lesinski M, Muehlbauer T, Granacher U. Concurrent validity of the Gyko inertial sensor system for the assessment of vertical jump height in female sub-elite youth soccer players. BMC Sports Sci Med Rehabil. 2016;8:35; doi: 10.1186/s13102-016-0061-x.

37. Magnúsdóttir Á, Porgilsson B, Karlsson B. Comparing three devices for jump height measurement in a heterogeneous group of subjects. J Strength Cond Res. 2014; 28(10):2837-2844; doi: 10.1519/JSC.000000000000 0464.

38. Mahmoud I, Othman AAA, Abdelrasoul E, Stergiou P, Katz L. The reliability of a real time wearable sensing device to measure vertical jump. Procedia Eng. 2015; 112:467-472; doi: 10.1016/j.proeng.2015.07.226.

39. Martínez-Martí F, González-Montesinos JL, Morales DP, Santos JRF, Castro-Piñero J, Carvajal MA, et al. Validation of instrumented insoles for measuring height in vertical jump. Int J Sports Med. 2016;37(5):374381; doi: 10.1055/s-0035-1565137.

40. Montoye AHK, Mitrzyk J. Validity of the Blast Athletic Performance monitor for assessing vertical jump height in female volleyball players. Meas Phys Educ Exerc Sci. 2019;23(2):99-109; doi: 10.1080/1091367X. 2018.1539739.

41. Rago V, Brito J, Figueiredo P, Carvalho T, Fernandes T, Fonseca $\mathrm{P}$, et al. Countermovement jump analysis using different portable devices: implications for field testing. Sports. 2018;6(3):91; doi: 10.3390/sports6030091.

42. Rantalainen T, Hesketh KD, Rodda C, Duckham RL. Validity of hip-worn inertial measurement unit compared to jump mat for jump height measurement in adolescents. Scand J Med Sci Sports. 2018;28(10): 2183-2188; doi: 10.1111/sms.13243.

43. Spangler R, Rantalainen T, Gastin PB, Wundersitz D. Inertial sensors are a valid tool to detect and consistently quantify jumping. Int J Sports Med. 2018;39(10):802808; doi: 10.1055/s-0044-100793.

44. Stanton R, Doering TM, Macgregor C, Borges N, Delvecchio L. Validity of a contact mat and accelerometric system to assess countermovement jump from flight time. Meas Phys Educ Exerc Sci. 2019;23(1):39-46; doi: 10.1080/1091367X.2018.1493593.

45. Toft Nielsen E, Jørgensen PB, Mechlenburg I, Sørensen $\mathrm{H}$. Validation of an inertial measurement unit to determine countermovement jump height. Asia Pac J Sports Med Arthrosc Rehabil Technol. 2019;16:8-13; doi: 10.1016/j.asmart.2018.09.002.

46. Watkins CM, Maunder E, van den Tillaar R, Oranchuk DJ. Concurrent validity and reliability of three ultra-portable vertical jump assessment technologies. Sensors. 2020;20(24):7240; doi: 10.3390/s20247240.

47. Zihajehzadeh S, Lee TJ, Lee JK, Hoskinson R, Park EJ. Integration of MEMS inertial and pressure sensors for vertical trajectory determination. IEEE Trans Instrum Meas. 2015;64(3):804-814; doi: 10.1109/TIM.2014. 2359813.

48. Wang J, Xu J, Shull PB. Vertical jump height estimation algorithm based on takeoff and landing identification via foot-worn inertial sensing. J Biomech Eng. 2018;140(3):034502; doi: 10.1115/1.4038740.

49. Borges TO, Moreira A, Bacchi R, Finotti RL, Ramos M, Lopes CR, et al. Validation of the VERT wearable jump monitor device in elite youth volleyball players. Biol Sport. 2017;34(3):239-242; doi:10.5114/biolsport.2017.66000.

50. Brooks ER, Benson AC, Bruce LM. Novel technologies found to be valid and reliable for the measurement of vertical jump height with jump-and-reach testing. J Strength Cond Res. 2018;32(10):2838-2845; doi: 10.1519/JSC.0000000000002790.

51. Casartelli N, Müller R, Maffiuletti NA. Validity and reliability of the Myotest accelerometric system for the assessment of vertical jump height. J Strength Cond Res. 2010;24(11):3186-3193; doi: 10.1519/JSC.0b013e318 1d8595c.

52. Choukou M-A, Laffaye G, Taiar R. Reliability and validity of an accelerometric system for assessing vertical jumping performance. Biol Sport. 2014;31(1):5562; doi: 10.5604/20831862.1086733.

53. Nickerson BS, Medrano NF, Perez GL, Narvaez SV, Carrillo J, Duque M. Inter-device reliability of wearable technology for quantifying jump height in collegiate athletes. Biol Sport. 2020;37(4):383-387; doi: 10.5114/ biolsport.2020.96851.

54. Nuzzo JL, Anning JH, Scharfenberg JM. The reliability of three devices used for measuring vertical jump height. J Strength Cond Res. 2011;25(9):2580-2590; doi: 10.1519/JSC.0b013e3181fee650.

55. Choukou MA, Laffaye G, Taiar R. Validity of an accelerometric system for measuring force-time-based data during jumping tasks. Comput Methods Biomech Biomed Engin. 2013;16(Suppl. 1):84-85; doi: 10.1080/ 10255842.2013.815849.

56. Silva R, Rico-González M, Lima R, Akyildiz Z, PinoOrtega J, Clemente FM. Validity and reliability of mobile applications for assessing strength, power, velocity, 


\section{HUMAN MOVEMENT}

F.M. Clemente et al., Inertial measurement units for jump height estimations

and change-of-direction: a systematic review. Sensors. 2021;21(8):2623; doi: 10.3390/s21082623.

57. Doğan NÖ. Bland-Altman analysis: a paradigm to understand correlation and agreement. Turk J Emerg Med. 2018;18(4):139-141; doi: 10.1016/j.tjem.2018.09.001.

58. Giavarina D. Understanding Bland Altman analysis. Biochem Med. 2015;25(2):141-151; doi: 10.11613/BM. 2015.015.

59. Akenhead R, Nassis GP. Training load and player monitoring in high-level football: current practice and perceptions. Int J Sports Physiol Perform. 2016;11(5):587593; doi: 10.1123/ijspp.2015-0331.

60. Guerrero-Calderón B, Klemp M, Castillo-Rodriguez A, Morcillo JA, Memmert D. A new approach for trainingload quantification in elite-level soccer: contextual factors. Int J Sports Med. 2021;42(8):716-723; doi: 10.1055/ a-1289-9059.

61. Lacome M, Simpson B, Broad N, Buchheit M. Monitoring players' readiness using predicted heart-rate responses to soccer drills. Int J Sports Physiol Perform. 2018;13(10):1273-1280; doi: 10.1123/ijspp.2018-0026.

62. Hopkins WG. Measures of reliability in sports medicine and science. Sports Med. 2000;30(1):1-15; doi: 10.2165/00007256-200030010-00001. 